\title{
Eletromiografia do músculo peitoral maior após reconstrução cirúrgica da ruptura crônica do tendão*
}

\section{Electromyography of the Pectoralis Major Muscle after Surgical Reconstruction of Chronic Tendon Rupture}

\author{
Benno Ejnisman ${ }^{1}$ Carlos Vicente Andreoli ${ }^{1}$ Paulo Santoro Belangero ${ }^{1}$ William Ricado Komatsu ${ }^{2}$ \\ Debora Cristina Hipolide ${ }^{3}$ Alberto de Castro Pochini ${ }^{10}$ \\ ${ }^{1}$ Departamento de Ortopedia e Traumatologia, Universidade Federal \\ de São Paulo, São Paulo, São Paulo, Brasil \\ 2 Departamento de Medicina do Esporte e Atividade Física, \\ Universidade Federal de São Paulo, São Paulo, São Paulo, Brasil \\ ${ }^{3}$ Departamento de Psicobiologia, Universidade Federal de São Paulo, \\ Address for correspondence Endereço para correspondência: Alberto \\ de Castro Pochini, Departamento de Ortopedia e Traumatologia, \\ Universidade Federal de São Paulo, Rua Estado de Israel, 636, Vila \\ Clementino, São Paulo, SP, 01421001, Brasil \\ (e-mail: apochini@uol.com.br).
} São Paulo, São Paulo, Brasil

Rev Bras Ortop 2021;56(1):31-35.

\section{Resumo \\ Palavras-chave \\ - músculos peitorais/lesões \\ - eletromiografia \\ - lesões atléticas \\ - levantamento de peso/lesões}

Objetivo Avaliar a atividade eletrofisiológica do músculo peitoral maior (PM) lesionado de pacientes operados que realizam halterofilismo, mais especificamente exercícios de supino, especialmente a atividade das porções clavicular e esternocostal do PM.

Métodos Todos os atletas no estudo I (10 pacientes) tiveram rupturas completas unilaterais durante o exercício de supino, e tinham histórico de uso de esteroides anabolizantes, associação descrita em até $86,7 \%$ das rupturas tendinosas do PM. O grupo controle incluiu 10 homens sem lesão no tendão do PM que não realizaram exercícios de supino. Descrição do projeto transversal. Os valores de $p$ foram obtidos por múltiplas comparações com a correção de Bonferroni.

Resultados Na comparação entre o grupo controle (C) e os halterofilistas durante o pós-operatório (POS), não foram encontradas diferenças nas medidas obtidas nas porções clavicular e esternocostal do músculo PM: nível médio clavicular $(p=0,847)$; desvio padrão (DP) clavicular $(p=0,777)$; área clavicular $(p=0,933)$; mediana da clavícula $(p=0,972)$; nível médio esternocostal $(p=0,633)$; DP esternocostal $(p=0,602)$; área esternocostal $(p=0,931)$; e mediana esternocostal $(p=0,633)$.

Conclusão Neste estudo, a atividade eletromiográfica do músculo PM em atletas de halterofilismo (exercício de supino) que foram submetidos a cirurgia esteve dentro dos parâmetros normais para as porções claviculares e esternocostais estudadas.

\footnotetext{
Estudo realizado no Departamento de Ortopedia e Traumatologia da Universidade Federal de São Paulo, São Paulo, Brasil.
}

recebido

27 de Janeiro de 2020

aceito

10 de Março de 2020
DOI https://doi.org/

10.1055/s-0040-1713387. ISSN $0102-3616$. (c) 2021. Sociedade Brasileira de Ortopedia e Traumatologia. All rights reserved.

This is an open access article published by Thieme under the terms of the Creative Commons Attribution-NonDerivative-NonCommercial-License, permitting copying and reproduction so long as the original work is given appropriate credit. Contents may not be used for commercial purposes, or adapted, remixed, transformed or built upon. (https://creativecommons.org/ licenses/by-nc-nd/4.0/)

Thieme Revinter Publicações Ltda., Rua do Matoso 170, Rio de Janeiro, RJ, CEP 20270-135, Brazil 


\author{
Abstract

\section{Keywords} \\ - pectoralis muscles/ \\ injuries \\ - electromyography \\ - athletic injuries \\ - weightlifting/ \\ injuries
}

Objectives To evaluate the electrophysiological activity of the injured pectoralis major (PM) muscle of operated patients who perform weightlifting, more specifically bench press exercises, especially the activity of the clavicular and sternocostal portions of the PM.

Methods All athletes in study I (10 patients) had unilateral complete ruptures during bench press exercises and a history of use of anabolic steroids, an association that is described in up to $86.7 \%$ of PM tendon ruptures. The control group included 10 men without PM tendon injury who did not perform bench press exercises. Description of the cross-sectional design. The $p$-values were obtained by multiple comparisons with Bonferroni correction.

Results In the comparison between the control (C) group and the weightlifters during the postoperative period (POS), we found no evidence of differences in any measurements obtained in the clavicular and sternocostal portions of the PM muscle: clavicular average level $(p=0.847)$; clavicular standard deviation (SD) $(p=0.777)$; clavicular area $(p=0.933)$; clavicular median $(p=0.972)$; sternocostal average level $(p=0.633)$; sternocostal SD $(p=0.602)$; sternocostal area $(p=0.931)$; and sternocostal median $(p=0.633)$.

Conclusion In the present study, the electromyographic activity of the PM muscle in weightlifters (bench press exercise) who underwent surgery was within the normal parameters for the clavicular and sternocostal portions studied.

\section{Introdução}

A ruptura do músculo peitoral maior (PM) tem se tornado cada vez mais comum devido à associação entre o uso de academia, uso de esteroides anabolizantes, e o sexo masculino (não há relatos de ruptura de PM em mulheres). ${ }^{1,2}$ No caso de frequentadores de academia ou atletas, estágios crônicos de lesão de PM podem resultar em perda significativa de adução (de $10 \%$ a $50 \%$ ) e importante deformidade cosmética do hemitórax. ${ }^{3-14}$

O tratamento cirúrgico tem sido recomendado nesses pacientes para restabelecer a função e a estética. ${ }^{2}$ Em geral, a porção esternocostal é tipicamente comprometida em atletas e fisiculturistas (lesão no supino). As lesões da porção clavicular do tendão do PM variam em termos da extensão do dano e do número de pacientes afetados, mas esse tipo de lesão é caracterizado por uma perda funcional de força residual que limita essa população de atletas e frequentadores de academia. ${ }^{9,10,14,15} \mathrm{O}$ retorno às atividades físicas regulares geralmente ocorre em mais de $90 \%$ dos pacientes (nossos estudos) após a reparação do tendão do PM e a reconstrução cirúrgica, que são grandes cirurgias que requerem o uso de enxerto tendinoso.

Mesmo com bons desfechos clínicos e funcionais durante o pós-operatório da cirurgia tendinosa do PM, persistem dúvidas sobre a atividade eletrofisiológica do músculo lesionado.

A eletromiografia (EMG) permite o registro extracelular da atividade bioelétrica gerada pelas fibras musculares. É realizada usando um eletrodo de superfície, que mede a atividade elétrica de várias unidades motoras ao mesmo tempo. ${ }^{16}$ Apesar de captar a atividade elétrica gerada pelo recrutamento das unidades motoras e não pela força muscular, ${ }^{17}$ a literatura sugere uma boa correlação entre o número de unidades motoras ativadas e a força muscular. Assim, esse método desempenha um papel importante na ilustração do perfil eletrofisiológico do músculo lesionado e reconstruído no momento do exame, e auxilia na evolução durante a fisioterapia e retorno ao esporte. Este estudo teve como objetivo avaliar o PM de pacientes operados que realizam halterofilismo, mais especificamente, exercícios de supino, especialmente a atividade das porções clavicular e esternocostal do PM.

Embora esse tipo de avaliação esteja sendo cada vez mais utilizado no cuidado clínico e na pesquisa científica, falta um consenso sobre vários aspectos do método. A colocação do sensor, o número de contrações de fibras fásicas, o tempo de contração de fibras tônicas, a necessidade de avaliação concomitante dos músculos sinérgicos, e a possibilidade de uso em situações especiais ainda devem ser padronizados.

\section{Materiais e Métodos}

\section{População do Estudo}

Foram analisados 20 atletas de halterofilismo previamente cadastrados e tratados no Centro de Traumatologia EsportivaOrtopedia, de acordo com o número 20959813.0.0000.5505 da Plataforma Brasil.

Todos os atletas do estudo I (10 pacientes) apresentaram rupturas completas unilaterais durante o exercício de supino e histórico de uso de esteroides anabolizantes, 
associação descrita em até $86,7 \%$ das rupturas tendinosas do PM. O grupo controle incluiu dez praticantes de exercícios de resistência sem lesão no tendão do PM que não realizaram exercícios de supino. Os indivíduos foram obrigados a assinar um termo de consentimento livre e esclarecido.

\section{Critérios de Inclusão}

\section{Estudo I}

\section{Grupo de Casos}

Esse grupo incluiu dez indivíduos que já estavam sendo monitorados no ambulatório de Medicina Esportiva, tinham histórico de ruptura do tendão peitoral e reconstrução cirúrgica do tendão do PM seguindo o protocolo padrão, ${ }^{14}$ realizaram exercícios de supino pelo menos três vezes por semana antes da lesão, tinham mais de dez anos de experiência competitiva de halterofilismo no supino, e tinham histórico de uso de esteroides anabolizantes.

\section{Grupo de Controle}

Este grupo incluiu dez indivíduos que eram compatíveis com sexo e idade em comparação com o grupo de casos, eram sedentários ou praticavam esportes esporadicamente, e não tinham histórico de uso de esteroides anabolizantes.

\section{Critérios de Exclusão}

Foram excluídos do estudo indivíduos com lesão tendinosa do PM ocorridas durante esportes que não fossem halterofilismo, indivíduos sem histórico de uso de esteroides, e atletas com histórico de doença crônica, como diabetes, nefropatia ou outras doenças que são conhecidas por apresentarem tendinopatia.

\section{Avaliações}

\section{Avaliação Clínica}

Todos os sujeitos responderam a um questionário específico avaliando o período em que realizaram o halterofilismo e o tipo e a frequência do uso de esteroides anabolizantes nos últimos 12 meses.

Em média, os pacientes com lesão crônica de PM tiveram um período de espera de 5,5 meses entre a lesão e a cirurgia de reconstrução do PM com a mesma técnica operatória. ${ }^{11} \mathrm{~A}$ técnica cirúrgica utilizada foi previamente descrita em nossos estudos, ${ }^{14}$ e o protocolo de reabilitação utilizado também foi padrão para este tipo de lesão e cirurgia.

\section{Eletromiografia}

Visitas ao Centro de Traumatologia Esportiva-Ortopedia foram agendadas por chamada telefônica, e os indivíduos foram submetidos à EMG, que foi coletada dinamicamente usando um MegaWin 3.1, ME-6000 T-8 canal, versão 3.0, com sistema com uma frequência de calibração de $2.000 \mathrm{~Hz}$, filtro de passe alto de $20 \mathrm{~Hz}$, e filtro de passe baixo de $500 \mathrm{~Hz}$.

Foram utilizados eletrodos Meditrace (DBI Medical, São Paulo, SP, Brasil) descartáveis, adesivos, passivos e monopolares, juntamente com gel sólido, de prata/cloreto de prata
$(\mathrm{Ag} / \mathrm{AgCl})$, com área de captura de $1 \mathrm{~cm}$, e distância de $2 \mathrm{~cm}$ entre os eletrodos. Os pacientes foram analisados durante os exercícios de supino e submetidos à EMG seguindo o protocolo validado, no qual os eletrodos foram colocados com base em medições de fita da clavícula para o processo xifoide, considerando $60 \%$ desse comprimento como área muscular do PM. Após a determinação desse valor, 80\% da largura do PM foram calculados medindo a inserção do PM do úmero para o esterno. O resultado para a largura de $80 \%$ foi considerado o ponto central, e um eletrodo foi colocado em cada lado desse ponto seguindo a direção das fibras musculares no lado dominante. $O$ eletrodo terra foi colocado na epífise medial da clavícula no lado dominante.

Os atletas realizaram uma série máxima de cada exercício com uma carga equivalente ao máximo de 10 repetições. A ordem dos exercícios foi aleatória entre os indivíduos. $\mathrm{O}$ exercício foi realizado utilizando-se equipamento de prensa olímpica da linha FW. Os praticantes foram instruídos a realizar a fase excêntrica dirigindo a barra em uma linha próxima ao centro do esterno, sem tocar no peito para evitar o movimento do eletrodo.

Um total de 20 atletas foram selecionados para medição eletromiográfica no exercício de supino para avaliar o recrutamento das duas principais porções do músculo PM durante o exercício realizado no pós-operatório de reconstrução tendinosa do PM utilizando enxertos tendinosos flexores.

Todos os pacientes crônicos foram avaliados por eletromiografia aos 5 meses de pós-operatório.

\section{Projeto de Estudo}

\section{Descrição do Projeto Transversal}

\section{Métodos de Análise}

As variáveis numéricas são descritas pela média e desvio padrão (DP), e as variáveis categóricas são descritas pelas frequências absoluta e relativa. Os modelos de equação de estimativa generalizada (EEG) foram ajustados considerando a dependência entre os lados do mesmo indivíduo. Os modelos foram equipados por uma função de distribuição gama e link de log, e os resultados são apresentados como os valores médios estimados e intervalos de confiança de $95 \%$. Os valores de $p$ foram obtidos por múltiplas comparações com a correção de Bonferroni.

As análises foram realizadas utilizando-se o programa Statistical Package for the Social Sciences (SPSS, IBM Corp., Armonk, NY, EUA), versão 19, e foi adotado um nível de significância de $5 \%$.

\section{Resultados}

As medidas das porções claviculares e esternocostais do músculo PM foram obtidas utilizando-se EMG bilateral em halterofilistas e controles para comparar os grupos; os lados direito e esquerdo foram considerados medidas de réplica de um sujeito.

Um paciente foi perdido no pós-operatório porque não retornou para a avaliação de seguimento de cinco meses. 
Tabela 1 Medições das porções claviculares e esternocostais do músculo peitoral maior obtidas por eletromiografia em controles saudáveis e em halterofilistas, após cirurgia, nos lados operado e contralateral

\begin{tabular}{|l|l|l|l|l|l|}
\hline & \multicolumn{2}{|l|}{ Grupos } & \multicolumn{2}{l|}{ Valor de $p$} \\
\hline & Controle (C) & $\begin{array}{l}\text { Pós-operatório: lado } \\
\text { operado (POS) }\end{array}$ & $\begin{array}{l}\text { Pós-operatório: lado } \\
\text { contralateral (POCL) }\end{array}$ & C x POS & $\begin{array}{l}\text { POS } \\
\text { x POCL }\end{array}$ \\
\hline Clavicular & & & & & \\
\hline Nível médio & $293,2(200,0,386,3)$ & $273,8(99,7,447,8)$ & $203,7(99,6,307,7)$ & 0,847 & 0,058 \\
\hline $\begin{array}{l}\text { Desvio } \\
\text { padrão }\end{array}$ & $104,9(70,3,139,5)$ & $96,1(46,1,146,1)$ & $72,4(35,3,109,6)$ & 0,777 & 0,002 \\
\hline Área & $7.481,4(4.896,9,10.065,9)$ & $7.743,2(2.230,2,13.256,2)$ & $5.773,1(2.429,0,9.117,2)$ & 0,933 & 0,09 \\
\hline Mediana & $269,9(174,5,365,2)$ & $266,0(76,4,455,6)$ & $194,7(90,2,299,2)$ & 0,972 & 0,109 \\
\hline Esternocostal & & & & & \\
\hline Nível médio & $345,0(204,0,486,0)$ & $304,4(216,0,392,9)$ & $233,2(160,3,306,2)$ & 0,633 & 0,121 \\
\hline $\begin{array}{l}\text { Desvio } \\
\text { padrão }\end{array}$ & $119,0(71,3,166,6)$ & $103,6(70,7,136,4)$ & $92,8(53,0,132,5)$ & 0,602 & 0,554 \\
\hline Área & $8.314,2(4.778,2,11.850,2)$ & $8.125,7(5.707,6,10543,8)$ & $6.471,4(4.200,6,8.742,3)$ & 0,931 & 0,126 \\
\hline Mediana & $335,9(187,8,484,0)$ & $292,4(193,4,391,5)$ & $215,2(143,7,286,8)$ & 0,633 & 0,091 \\
\hline
\end{tabular}

Notas: Os dados são expressos em $\mu \mathrm{V}$ como meio de estimativas e intervalos de confiança de 95\%; p: múltiplas comparações entre grupos.

Dos pacientes submetidos à cirurgia, foram avaliados 9 halterofilistas que realizaram exercícios de supino, tiveram lesão unilateral, e tinham idade média de 36,7 anos ( $D P=9,1$ anos). Foram analisados nove pacientes de controle para uma amostra homogênea entre o grupo $\mathrm{C}$ e nove casos. Além disso, foram avaliados nove pacientes de controle. As medidas foram obtidas pelo EMG nos lados lesionados submetidos a reconstrução do PM com enxerto de tendão flexor (grupo pós-operatório [POS]: nove medidas), e nas laterais contralaterais aos lados operados (grupo pós-operatório contralateral [POCL]: nove medidas).

As comparações entre os grupos foram realizadas por meio de modelos de montagem considerando a dependência entre as medições bilaterais de um mesmo indivíduo.

Na comparação entre os grupos C e POS, não foram encontradas diferenças ( - Tabela $\mathbf{1}$ ) em quaisquer medidas obtidas nas porções clavicular e esternocostal do músculo PM: nível médio clavicular $(p=0,847)$; DP clavicular $(p=0,777)$; área esternovicular $(p=0,933)$; mediana da clavícula $(p=0,972)$; nível médio esternocostal $(p=0,633)$; DP esternocostal ( $p=0,602)$; área esternocostal $(p=0,931)$; e mediana esternocostal $(p=0,633)$.

\section{Discussão}

A frequência da lesão muscular do PM levou a estudos da capacidade do músculo PM reparado ou cirurgicamente reconstruído de retornar à atividade funcional adequada. ${ }^{2-15}$

A avaliação isocinética utilizando o pico de torque e o trabalho muscular na adução horizontal tem sido muito útil na obtenção de uma avaliação mais objetiva, ainda que indireta, da força muscular do PM nos períodos pré e pós-operatório de lesões crônicas que requerem reconstrução do PM. Em geral, após a melhora, o nível de força muscular deve apresentar um déficit não superior a $15 \%$ do músculo contralateral. No entanto, essa melhora se deve ao recrutamento da atividade de partes musculares que não a parte esternocostal mais machucada? Este estudo ajuda a compreender melhor esses aspectos funcionais durante o pós-operatório nesses pacientes.

Uma das principais variáveis analisadas pela EMG é a contração voluntária máxima (CVM), que é realizada por fibras musculares de contração rápida (tipo II), e é responsável pela força muscular. ${ }^{1}$

Nos atletas submetidos à reconstrução do PM, a atividade EMG do músculo PM não foi diferente entre os lados lesionado e contralateral, o que pode indicar que o músculo reconstruído tem capacidade funcional para auxiliar na atividade de halterofilismo. Estudos de anatomia patológica não mostraram degeneração muscular significativa, mesmo em casos crônicos, de dois a cinco anos após a lesão do PM.

A maior atividade de EMG no lado operado na porção clavicular em relação à porção contralateral pode estar relacionada à tentativa da porção muscular não afetada pela ruptura de auxiliar a porção esternocostal lesionada. Assim, observa-se uma variância na melhora funcional.

Esses pacientes não foram submetidos à avaliação isocinética porque a recuperação do nível de força entre cinco meses e um ano após a cirurgia foi bem estabelecida em outros estudos publicados pelo nosso grupo de pesquisa e outros autores. A recuperação muscular é obviamente variável, mas, em média, permite um retorno suficiente à atividade competitiva.

Conforme descrito, o principal objetivo deste estudo foi examinar a atividade elétrica e funcional da contração muscular da musculatura lesionada e a musculatura da região clavicular. Em média, os pacientes tiveram um período de espera de 5,5 meses entre a lesão e a cirurgia de reconstrução do PM.

Todos os pacientes crônicos foram avaliados por eletromiografia aos cinco meses de pós-operatório. 
É possível que o tempo entre o exame de eletromiografia, a lesão, ou o exame pós-operatório possa ter algum impacto no resultado. Em nosso estudo, o tempo médio entre a lesão e a cirurgia foi de 5,5 meses. Os exames de eletromiografia foram realizados a cada cinco meses após a cirurgia.

\section{Conclusão}

Neste estudo, a atividade eletromiográfica do músculo PM em halterofilistas (exercício de supino) que foram submetidos a cirurgia esteve dentro dos parâmetros normais para as porções claviculares e esternocostais estudadas.

\section{Apoio Financeiro}

Este estudo foi apoiado pela Fundação de Amparo à Pesquisa de São Paulo (Fapesp) - processo 2014/04180-6.

\section{Financiamento}

Fapesp: http://dx.doi.org/10.13039/5011000018072014/ 04180-6 benno ejnisman.

\section{Conflito de Interesses}

Os autores declaram não haver conflito de interesses.

\section{Referências}

1 Auchincloss CC, McLean L. The reliability of surface EMG recorded from the pelvic floor muscles. J Neurosci Methods 2009;182(01): 85-96

2 Bak K, Cameron EA, Henderson IJ. Rupture of the pectoralis major: a meta-analysis of 112 cases. Knee Surg Sports Traumatol Arthrosc 2000;8(02):113-119

3 de Castro Pochini A, Andreoli CV, Belangero PS, et al. Clinical considerations for the surgical treatment of pectoralis major muscle ruptures based on 60 cases: a prospective study and literature review. Am J Sports Med 2014;42(01):95-102

4 de Castro Pochini A, Ejnisman B, Andreoli CV, et al. Exact moment of tendon of pectoralis major muscle rupture captured on video. Br J Sports Med 2007;41(09):618-619, discussion 619
5 de Castro Pochini A, Ejnisman B, Andreoli CV, et al. Pectoralis major muscle rupture in athletes: a prospective study. Am J Sports Med 2010;38(01):92-98

6 Ejnisman B, Andreoli CV, de Castro Pochini A, Carrera EF, Abdalla RJ, Cohen M. Ruptura do musculo peitoral maior em atletas. Rev Bras Ortop 2002;37(11):482-488

7 de Figueiredo EA, Terra BB, Cohen C, et al. Footprint do tendão do peitoral maior: estudo anatômico. Rev Bras Ortop 2013;48(06): 519-523

8 Fleury AM, Silva AC, de Castro Pochini A, Ejnisman B, Lira CA, Andrade MdosS. Isokinetic muscle assessment after treatment of pectoralis major muscle rupture using surgical or non-surgical procedures. Clinics (São Paulo) 2011;66(02):313-320

9 Lee J, Brookenthal KR, Ramsey ML, Kneeland JB, Herzog R. MR imaging assessment of the pectoralis major myotendinous unit: an MR imaging-anatomic correlative study with surgical correlation. AJR Am J Roentgenol 2000;174(05):1371-1375

10 Ohashi K, El-Khoury GY, Albright JP, Tearse DS. MRI of complete rupture of the pectoralis major muscle. Skeletal Radiol 1996;25 (07):625-628

11 Pochini A, Ejnisman B, Andreoli CV, Cohen M. Reconstruction of the pectoralis major tendon using autologous grafting and cortical button attachment: description of the technique. Tech Shoulder Elbow Surg 2012;13(05):77-80

12 Pochini AdeC, Andreoli CV, Ejnisman B, Maffulli N. Surgical repair of a rupture of the pectoralis major muscle. BMJ Case Rep 2015; 2015:bcr201320229

13 Pochini AdeC, Ferretti M, Kawakami EF, et al. Analisys of pectoralis major tendon in weightlifting athletes using ultrasonography and elastography. Einstein (Sao Paulo) 2015;13(04):541-546

14 Pochini AC, Rodrigues MSB, Yamashita L, Belangero PS, Andreoli CV, Ejnisman B. Surgical treatment of pectoralis major muscle rupture with adjustable cortical button. Rev Bras Ortop 2017;53(01):60-66

15 ElMaraghy AW, Devereaux MW. A systematic review and comprehensive classification of pectoralis major tears. J Shoulder Elbow Surg 2012;21(03):412-422

16 Vodusek DB. Electromyography. In: Bø K, Berghmas B, Mørkved S, Van Kampen M, editors. Evidence-based physical therapy for the pelvic floor. Philadelphia: Elsevier; 2007:53-63

17 Vodusek DB. The role of electrophysiology in the evaluation of incontinence and prolapse. Curr Opin Obstet Gynecol 2002;14 (05):509-514 